\title{
Intraobserver and Interobserver Agreement in the Scoring of PD-L1 (SP142) and Tumor-Infiltrating Lymphocytes in Triple Negative Breast Cancers*
}

\author{
David Jerome Ong, Pier Angeli Medina, Sarah Jane Datay-Lim, Elizabeth Ann Alcazaren
}

Department of Laboratory Medicine and Pathology, The Medical City, Pasig City, Philippines

\section{ABSTRACT}

Objectives. Known for their poor outcomes, triple negative breast cancers (TNBCS) have been investigated for immune checkpoint inhibitors that target Programmed death ligand 1 (PD-L1). In the recent decade, tumor-infiltrating lymphocytes (TILS) have also become potential biomarkers. The aim of the study is to determine the reproducibility of PD-L1 scoring system for TNBC (SP142 clone) and TILs interpretation in the local setting through intra- and interobserver agreement.

Methodology. Forty-three primary resection specimens TNBC were evaluated on two occasions with PD-L1 (Roche VENTANA SP142 assay) and TILs by two breast pathologists and one general pathologist on physical glass slides. PD-L1 expression was determined by at least $1 \%$ positivity among immune cells within the tumoral area and contiguous peritumoral stroma while TILs was assessed based on International ImmunoOncology Biomarker Working Group on Breast Cancer. Kappa statistic for PD-LI and TILs categories while intraclass correlation coefficient (ICC) were assessed, with cutoffs of 0.80 and 0.70 , respectively.

Results. The overall interrater kappa statistic for PD-L1 on the first and second rounds were weak at 0.506 (95\% Cl: 0.334-0.679) and minimal at 0.314 (95\% Cl: 0.142-0.487), respectively. Intraobserver kappa statistic for PD-L1 were varied across the three readers while interobserver kappa values for PD-L1 showed none $(0.181)$ to moderate $(0.789)$ agreement. The TILs intraobserver reliability showed poor to good agreement, with the highest ICC of 0.889 (95\% Cl: 0.805-0.938).

Conclusion. This study demonstrated variable intra and interobserver agreement for both TILS and PD-L1 expression. Although it is desirable to have strong to almost perfect agreement, the kappa and ICC values suggest additional room for improvement. In light of the repercussions in management of patients who will undergo immune checkpoint inhibitor therapy, regular training sessions, concurrences of equivocal results, and possible use of digital pathology as a medium in interpreting TILs and PD-L1 stains to achieve consistent results.

Key words: breast cancer, triple negative breast cancer, immunohistochemistry, PD-Ll, TILs

ISSN 2507-8364 (Online)

Printed in the Philippines.

Copyright $\odot 2021$ by the PJP.

Received: 11 October 2021.

Accepted: 13 November 2021.

Published online first: 9 December 2021.

https://doi.org/10.21141/PJP.2021.16

Corresponding author: David Jerome P. Ong, MD, MBA

E-mail:d_p_ong@yahoo.com

ORCiD: https://orcid.org/0000-0002-4204-3223

* Presented as e-poster at the $60^{\text {th }}$ IAP-Thailand Annual Meeting 2021 at Bangkok, Thailand, October 6-8, 2021.

\section{INTRODUCTION}

Breast cancer still remains as the number one reported cancer. In 2020, there are 27,163 cases of newly diagnosed breast cancer $(17.7 \%)$ in the Philippines. ${ }^{1}$ Triple negative breast cancers (TNBC), by definition, lack the expression for estrogen receptor (ER), progesterone receptor (PR) and HER2/neu using immunohistochemistry (IHC) stains. TNBCs are known for their poor outcomes and are unresponsive to conventional hormonal therapy. ${ }^{2}$ Tumor-infiltrating lymphocytes (TILs) were reported to have prognostic value in TNBC and are used as a clinical biomarker for the prognostication of TNBC. Higher TILs were regarded as good prognostic indicators for patients who receive atezolizumab monotherapy. ${ }^{3}$

Programmed death ligand 1 (PD-L1) found on the surface of some inflammatory cells has a role in the downregulation of the immune pathway leading to reduced production of cytokines, increasing tumorigenesis and tumor aggressiveness. ${ }^{4}$ In addition, PD-L1 positivity was seen to be associated with increased stromal TILs and improved survival. Both PD-L1 interpretation and TILs require 
necessary experience and training prior to becoming the standard of histopathological reports of breast carcinoma. ${ }^{5}$

Reporting of TILs have been encouraged as part of the histopathological examination of breast carcinomas, spearheaded by the International Immuno-Oncology Biomarker Working Group on Breast Cancer and mentioned in the 2019 WHO Classification of Breast Tumors. ${ }^{6,7}$ However, its implementation as part of routine practice requires validation, which includes testing its reproducibility. To date, there are limited studies on intraobserver and interobserver variability in the local setting. PD-L1 is a biomarker for TNBC, and it would be a good measure to evaluate its precision and applicability among Filipino pathologists. It can also be used as a measure of service quality within the laboratory by looking into the consistency and accuracy of rendered results. Hence, this study aimed to determine the reliability of PD-L1 and TILs scoring.

\section{METHODOLOGY}

\section{Case Selection}

This study was approved by The Medical City Institutional Review Board. All TNBC patient specimens from 20172019 were identified using the laboratory information system (Technidata) of the Section of Anatomic Pathology, Department of Laboratory Medicine and Pathology. The specimen selection criteria were as follows: resection specimens confirmed to have TNBC by IHC stains from 2017 to 2019. Patients with equivocal or positive HER2 IHC stains, DCIS-only by histopathologic diagnosis were excluded. Out of 100 TNBC from 2017-2019, 43 resection specimens were included in the study. None of the specimens were metastatic TNBC. Clinicopathologic information such as age, sex, tumor size, tumor focality, histologic type, Nottingham histologic grade, nuclear grade, pathologic stage, lymph node metastasis, lymphovascular invasion, presence or absence of DCIS, previous neoadjuvant chemotherapy was obtained from medical records and the laboratory information system.

\section{Readers}

Three pathologists were identified as part of the study. Their expertise varied from three to more than 30 years of experience in anatomic pathology. Two out of three pathologists had prior clinical observership in breast pathology. In terms of PD-L1 interpretation, one had prior experience with PD-L1 SP263 clone, but none had PD-L1 any previous sign-out with the SP142 clone. All pathologists underwent training using the Roche Tissue Diagnostics Pathology Education Portal for PD-L1 (SP142) TNBC Interpretation Education and were subsequently certified. On the other hand, no online training for TILs scoring was available as of this writing. The readers familiarized with the TILs working group consensus guidelines and used reference images from their website.

\section{PD-L1 Scoring and TIL Evaluation}

The IHC stains were performed on paraffin embedded tissues fixed in $10 \%$ neutral buffered formalin for 6-72 hours. All chosen tissues were stained with Roche VENTANA PDL1 (SP142) Assay antibody detection kit using BenchMark ULTRA System (Ventana Medical Systems, Inc.) by one proficient registered medical technologist. Pre-analytic factors such as positive and negative controls, adequacy of specimen (at least 50 viable invasive tumor cells) and tissue processing were considered prior to scoring PD-L1 ${ }^{8}$ The hematoxylin and eosin (H\&E) stained slides and previous ER, PR and HER2 IHC stains were evaluated alongside the PD-L1 stained slides. Positivity for PD-L1 expression was determined by a cut-off of $1 \%$ among immune cells with dark brown punctate, linear, or circumferential staining within the tumoral area and contiguous peritumoral stroma. Conversely, a score below $1 \%$ is considered negative. ${ }^{8}$ TIL scoring was performed on the H\&E stained slide based on the recommendations of International Immuno-Oncology Biomarker Working Group on Breast Cancer. Evaluation of the stromal area within the defined tumor borders for mononuclear infiltrates were done using low-power magnification (10X). Percentage of stromal lymphocytes were reported as averages of the entire area evaluated. TILs were reported as a continuous variable, as recommended by the TILs working group. ${ }^{6}$ For this study, interpretations were categorized arbitrarily as follows: low (0-10\%), intermediate (11-50\%), high (>50\%), following reproducibility studies on TILs. ${ }^{9,10}$ Interpretation was performed on two separate occasions following at least a two-week washout period. The sequence of control numbers was randomized, and the pathologists were blinded to the results of their previous interpretations.

\section{Analysis}

Descriptive statistics of the demographic, histopathological variables of the case selection were determined using frequency and percentage for categorical variables and mean and standard deviation for continuous data. PDL1 expression and categorical TILs group were also reported in frequencies, by each reader. Intra- and interobserver agreement for interpretation of PD-L1 was determined using Cohen's kappa while overall agreement was determined using Fleiss's Kappa. Interpretation of kappa for PD-L1 was based on the proposed ranges of McHugh: 0.00-0.20 as none, 0.21-0.39 as minimal, 0.400.59 as weak, $0.60-0.79$ as moderate, $0.80-0.90$ as strong, and above 0.90 as almost perfect agreement. This is more stringent than Cohen's original suggestion for interpreting kappa, with larger ranges. As the value of kappa decreases, the percent reliability of data decreases due to increasing disagreement among readers. Hence, interpretation of kappa must be done in the appropriate clinical and laboratory context. ${ }^{11}$ An acceptable kappa for PD-L1 would be 0.80 and above with McHugh's kappa interpretation to ensure quality results in the clinical laboratory setting.

On the other hand, intra- and interobserver agreement for TILs reported as categorical data were determined using weighted kappa. For TILs reported as continuous data, the Intraclass Correlation Coefficient (ICC) as single measures was used to determine the agreement among three readers. While there are no standard values for interpreting ICC, an ICC of 0.7 is considered acceptable among three readers with a $95 \%$ CI. Interpretation of the ICC, as recommended by $\mathrm{Koo}$ and $\mathrm{Li}$, were as follows: less than 0.50 as poor, $0.50-0.75$ as moderate, $0.75-0.90$ as good and greater than 0.90 as excellent reliability. ${ }^{12}$ Statistical Package for the Social Sciences (SPSS) version 20 was used in the analysis. 


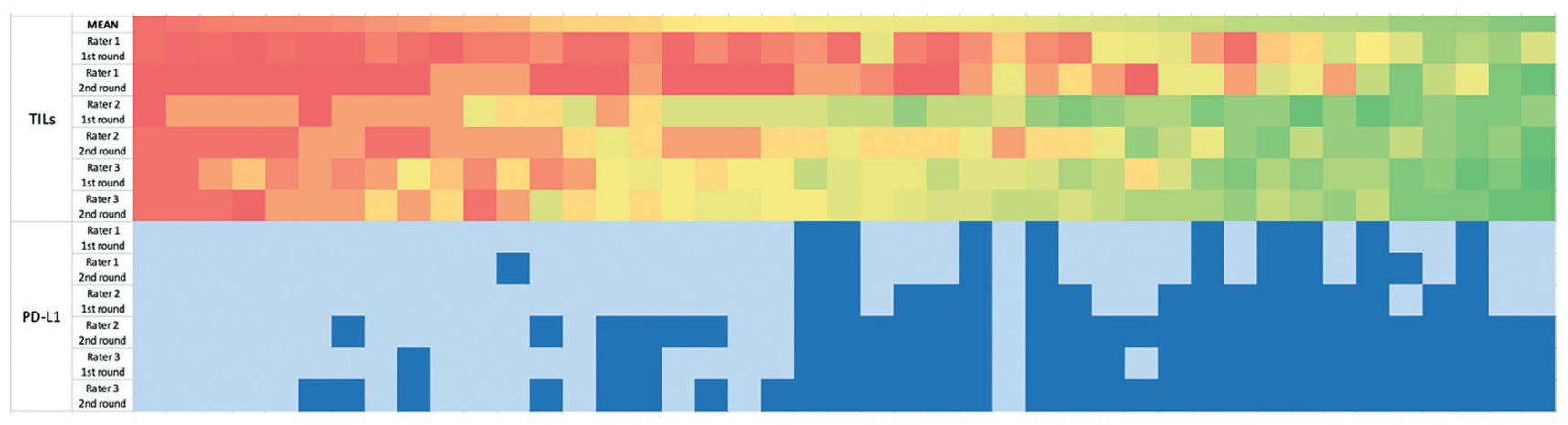

Figure 1. Heat map of the individual interpretations of TILs and PD-L1 arranged according to mean on the topmost row.

\section{RESULTS}

Among 1039 samples tested for ER, PR and HER2 from 2017-2019, 100 specimens (9.62\%) were TNBCs and only 43 were resection specimens that were included in the study. Core biopsies were excluded The most frequent tumors were invasive breast carcinoma of no special type (IDCA) including medullary pattern $(67.4 \%)$, followed by invasive lobular carcinoma (16.3\%) and metaplastic carcinoma $(7.0 \%)$, and other entities (16.3\%). There were nine cases that had neoadjuvant chemotherapy (Table 1).

Table 1. Baseline clinical characteristics of triple negative breast cancer and PD-L1 expression in a tertiary hospital

\begin{tabular}{|c|c|c|}
\hline Clinical characteristics & $\mathbf{n}$ & $\%$ \\
\hline Age, year mean (SD) & $50.9( \pm 11.73)$ & $11.7 \%$ \\
\hline $20-39$ years & 7 & $16.3 \%$ \\
\hline 40-59 years & 28 & $65.1 \%$ \\
\hline 60 years and above & 8 & $18.6 \%$ \\
\hline Sex (Female) & 43 & $100.0 \%$ \\
\hline Tumor size, cm mean (SD) & 4.1 & $3.5 \%$ \\
\hline \multicolumn{3}{|l|}{ Tumor focality } \\
\hline Single & 35 & $81.4 \%$ \\
\hline Multiple & 8 & $18.6 \%$ \\
\hline \multicolumn{3}{|l|}{ Histologic type } \\
\hline Invasive breast carcinoma of no special type & 29 & $67.4 \%$ \\
\hline Invasive lobular carcinoma and related entities & 7 & $16.3 \%$ \\
\hline Metaplastic carcinoma and other subtypes & 3 & $7.0 \%$ \\
\hline Microinvasive carcinoma & 3 & $7.0 \%$ \\
\hline Mucinous carcinoma & 1 & $2.3 \%$ \\
\hline \multicolumn{3}{|l|}{ Nottingham Histologic Grade } \\
\hline I & 6 & $15.0 \%$ \\
\hline II & 24 & $60.0 \%$ \\
\hline III & 10 & $25.0 \%$ \\
\hline \multicolumn{3}{|l|}{ Nuclear grade } \\
\hline 1 & 4 & $9.3 \%$ \\
\hline 2 & 24 & $55.8 \%$ \\
\hline 3 & 15 & $34.9 \%$ \\
\hline Lymph node metastasis & 16 & $37.2 \%$ \\
\hline Lymphovascular invasion & 20 & $46.5 \%$ \\
\hline Presence of DCIS & 23 & $53.5 \%$ \\
\hline Necrosis in DCIS & 16 & $37.2 \%$ \\
\hline Neoadjuvant therapy & 9 & $20.9 \%$ \\
\hline
\end{tabular}

Individual scores for both PD-L1 expression and TILs are visually represented in a heat map, arranged by the mean TILs score from the three readers (Figure 1). Table 2 shows the reported frequencies of PD-L1 expression and TILs by each reader. Most of the TILs were rated as low, most especially by the first reader. PD-L1 disagreements occurred in nine and eight out of 43 cases in the first and second rounds, respectively, while TILs disagreements on the categorical level occurred in 28 and 25 out of the 43 cases in the first and second rounds, respectively.

The overall kappa statistic for PD-L1 on the first and second rounds were weak at 0.506 (95\% CI: 0.334-0.679) and minimal at 0.314 (95\% CI: 0.142-0.487), respectively. Intraobserver kappa statistic for PD-L1 were varied across the three readers: 0.482 (weak), 0.707 (moderate) and 0.870 (strong) (Table 3). Interobserver kappa values for PD-L1 showed a wide range of values from none to moderate agreement, with the lowest values belonging to reader 1 vs reader $3(\mathrm{k}=0.181)$ on the second round, and reader 1 vs reader $2(\mathrm{k}=0.230)$ on the second round. Moderate agreement was observed between reader 2 and reader 3 on both occasions $(\mathrm{k}=0.639, \mathrm{k}=0.789)$ (Table 4$)$.

In terms of categorical TILs, the overall ICC was weak at 0.494 (95\% CI: $0.149-0.720)$ and moderate at 0.667 (95\% CI: 0.391-0.821) on the first and second rounds, respectively. The intraobserver reliability showed moderate to good agreement, with the highest ICC of $0.889(95 \%$ CI: $0.805-0.938$ ) in terms of quantitative TILs and highest weighted kappa of 0.723 (95\% CI: 0.551-0.895) for categorical TILs, both belonging to reader 3 (Table 3). ICCs between each reader were tabulated in Table 4 . ICCs generally showed poor to good agreement (0.281 to 0.825 ). Good agreement was noted to be between readers 2 and 3 on both occasions. Of special note is the medullary pattern of IDCA (5/43 cases), wherein the TILs scoring ranged from low to high $(0-80 \%)$ and the PD-L1 was rated positive on 21 out of 30 occasions (for both rounds).

\section{Table 2. Frequency of PD-L1 and TILs interpretation among three readers}

\begin{tabular}{|c|c|c|c|c|c|c|}
\hline & \multicolumn{2}{|c|}{ Reader 1} & \multicolumn{2}{|c|}{ Reader 2} & \multicolumn{2}{|c|}{ Reader 3} \\
\hline & Round $1 \%$ & Round $2 \%$ & Round $1 \%$ & Round $2 \%$ & Round $1 \%$ & Round $2 \%$ \\
\hline PD-L1 $\geq 1 \%$ & 20.9 & 25.6 & 37.2 & 34.9 & 44.2 & 30.2 \\
\hline PD-L1 <1\% & 79.1 & 74.4 & 62.8 & 65.1 & 55.8 & 69.8 \\
\hline TILs - Low (0-10\%) & 74.4 & 76.7 & 32.6 & 60.5 & 34.9 & 32.6 \\
\hline TILs - Intermediate (11-50\%) & 20.9 & 16.3 & 32.6 & 20.9 & 46.5 & 51.2 \\
\hline TILs - High (>50\%) & 4.7 & 7.0 & 34.8 & 18.6 & 18.6 & 16.3 \\
\hline
\end{tabular}


Table 3. Intraobserver agreement of PD-L1 and TILs

\begin{tabular}{|c|c|c|c|c|c|c|}
\hline & \multicolumn{2}{|l|}{$\begin{array}{r}\text { PD-L1 } \\
\end{array}$} & \multicolumn{4}{|c|}{ TILs } \\
\hline & Kappa $(95 \% \mathrm{Cl})$ & $p$-value & Kappa $(95 \%$ CI) & $p$-value & ICC (95\% CI) & $p$-value \\
\hline Reader 1 & $0.870(0.696-1.000)$ & $<0.001$ & $0.418(0.134-0.701)$ & $<0.001$ & $0.666(0.462-0.804)$ & $<0.001$ \\
\hline Reader 2 & $0.482(0.269-0.695)$ & $<0.001$ & $0.449(0.269-0.629)$ & $<0.001$ & $0.629(0.212-0.820)$ & $<0.001$ \\
\hline Reader 3 & $0.707(0.500-0.915)$ & $<0.001$ & $0.723(0.551-0.895)$ & $<0.001$ & $0.889(0.805-0.938)$ & $<0.001$ \\
\hline
\end{tabular}

Table 4. Interobserver agreement of PD-L1 and TILS

\begin{tabular}{|c|c|c|c|c|c|c|}
\hline \multirow[b]{2}{*}{ 1st Round } & \multicolumn{2}{|c|}{ PD-L1 } & \multicolumn{4}{|c|}{ TILs } \\
\hline & Kappa $(95 \% \mathrm{Cl})$ & $p$-value & Kappa $(95 \% \mathrm{Cl})$ & $p$-value & ICC $(95 \% \mathrm{CI})$ & $p$-value \\
\hline 1 vs 2 & $0.618(0.378-0.857)$ & $<0.001$ & $0.225(0.090-0.360)$ & 0.003 & $0.281(-0.093-0.587)$ & $<0.001$ \\
\hline 2 vs 3 & $0.639(0.428-0.849)$ & $<0.001$ & $0.690(0.522-0.858)$ & $<0.001$ & $0.812(0.615-0.904)$ & $<0.001$ \\
\hline 1 vs 3 & $0.347(0.145-0.549)$ & 0.003 & $0.228(0.059-0.397)$ & 0.007 & $0.417(-0.040-0.698)$ & $<0.001$ \\
\hline 2nd Round & Kappa $(95 \% \mathrm{Cl})$ & $p$-value & Kappa $(95 \% \mathrm{Cl})$ & $p$-value & ICC $(95 \% \mathrm{CI})$ & $p$-value \\
\hline 1 vs 2 & $0.230(0.037-0.423)$ & 0.037 & $0.379(0.170-0.588)$ & $<0.001$ & $0.612(0.299-0.789)$ & $<0.001$ \\
\hline 2 vs 3 & $0.789(0.593-0.984)$ & $<0.001$ & $0.500(0.328-0.671)$ & $<0.001$ & $0.825(0.626-0.913)$ & $<0.001$ \\
\hline 1 vs 3 & $0.181(0.005-0.358)$ & 0.077 & $0.289(0.117-0.461)$ & $<0.001$ & $0.565(-0.022-0.814)$ & $<0.001$ \\
\hline
\end{tabular}

\section{DISCUSSION}

The prevalence of TNBCs in our institution is consistent with epidemiologic data of TNBCs, ranging from 10-20\% of all invasive breast carcinomas. ${ }^{13}$ The potential of PDL1 as a biomarker in patients with TNBC was investigated on the IMpassion 130 trial, as well as other studies, where overall survival benefit was suggested with the inclusion of an immune checkpoint inhibitor plus paclitaxel in patients with PD-L1 expression. ${ }^{14,15}$ Other anti-PD-L1 antibodies have been studied, namely durvalumab and avelumab, with similar molecular mechanisms. ${ }^{16}$ However, overall survival benefit of PD-L1 expression in a metaanalysis showed that breast cancer patients with increased PD-L1 expression led to poorer outcomes. A higher PD-L1 expression has been associated with lymph node metastasis, higher histologic grade and negative ER. All of which are linked to lower overall survival. ${ }^{17}$ In spite of this, immune checkpoint inhibitors may still have overall benefit given the limited data available to date. Moreover, TIL scoring is prognostic for TNBC in the context of PD-L1 expression. It is an emerging prognostic marker that has been recommended by the International Immuno-Oncology Biomarker Working Group on Breast Cancer and published in the 2019 WHO Classification of Breast Tumours. ${ }^{6}$ CD20+ TILs and PD-L1+ TILs were seen as independent prognostic factors for both TNBC and inflammatory breast cancer. ${ }^{18}$ TILs have been the target of interest for oncologists because of its potential as a biomarker, a predictive marker and a marker for targeted therapies. ${ }^{19-21}$

Eligibility for PD-L1 (SP142) immune checkpoint inhibitors requires a positivity of $\geq 1 \%$, a very low cutoff for positivity. PD-L1 may stain both tumor cells and tumor-infiltrating immune cells, which can be a source of confusion. The pattern of staining expected among immune cells should be dark brown, punctate, sometimes circumferential staining, especially for macrophages or dendritic cells. Immune cells may be stain singly or as aggregates. On the other hand, tumor cells can exhibit moderate to strong linear or circumferential stain. It is therefore required for the pathologist to compare the H\&E staining to allow distinction between tumor and immune cells. ${ }^{8}$
The laboratory must ensure that the issued result is validated and precise. In the case of PD-L1 expression, an agreement of less than 0.80 raises concerns on reproducibility. PD-L1 scoring has been subjected to various scrutiny because of its variability in interpretation. Intraand interobserver agreement in this study showed varied results, with modest agreement. Studies on interobserver variability of PD-L1 expression on TNBCs are mixed, with one showing high intraobserver and interobserver agreement while some show low reproducibility of SP142 assays.,22 Among antibody clones, the SP142 antibody showed the lowest interobserver agreement, but had substantial intraobserver agreement $(\mathrm{k}=0.798$ and 0.861 ), with the authors concluding that it is a reproducible interpretation..$^{23}$ On the other hand, reproducibility of the PD-L1 scoring was put into question in a study consisting of 19 pathologists across 14 different institutions. As the number of observers increased, the overall percent agreement decreased. The implications of this study is considerable in that some patients may or may not receive the proper treatment given the differences in scoring. ${ }^{21,22}$ Disagreements in PD-L1 expression in terms of categorical TILs showed no specific predilection, as illustrated by the heatmap in Figure 1. Hence, differences in interpreting PD-L1 were not necessarily affected by the amount stromal TILs present in the specimen.

Standard protocols for TIL scoring were proposed by the TILs Working Group using H\&E-stained slides with no need for ancillary procedures. No designated cutoffs on agreement have been proposed to date, and acceptable intra- and interpersonal agreement would depend on the institution or clinical use. ${ }^{6}$ Similar studies on TILs concordance have been published. In one study, an ICC cutoff of 0.70 for concordance of TIL assessment was deemed acceptable. Though not statistically significant, the authors concluded that TILs interpretation was reproducible but required further refinement and is yet to be implemented in the clinical setting. Specimen factors which may lead to incorrect reading of TILs include apoptosis, individual cell necrosis and stromal fibroblasts. ${ }^{10}$ Other factors were presence of reactive plasma cells mimicking tumor cells, plasmacytoid tumor cells mimicking infiltrating plasma cells, and specimens 

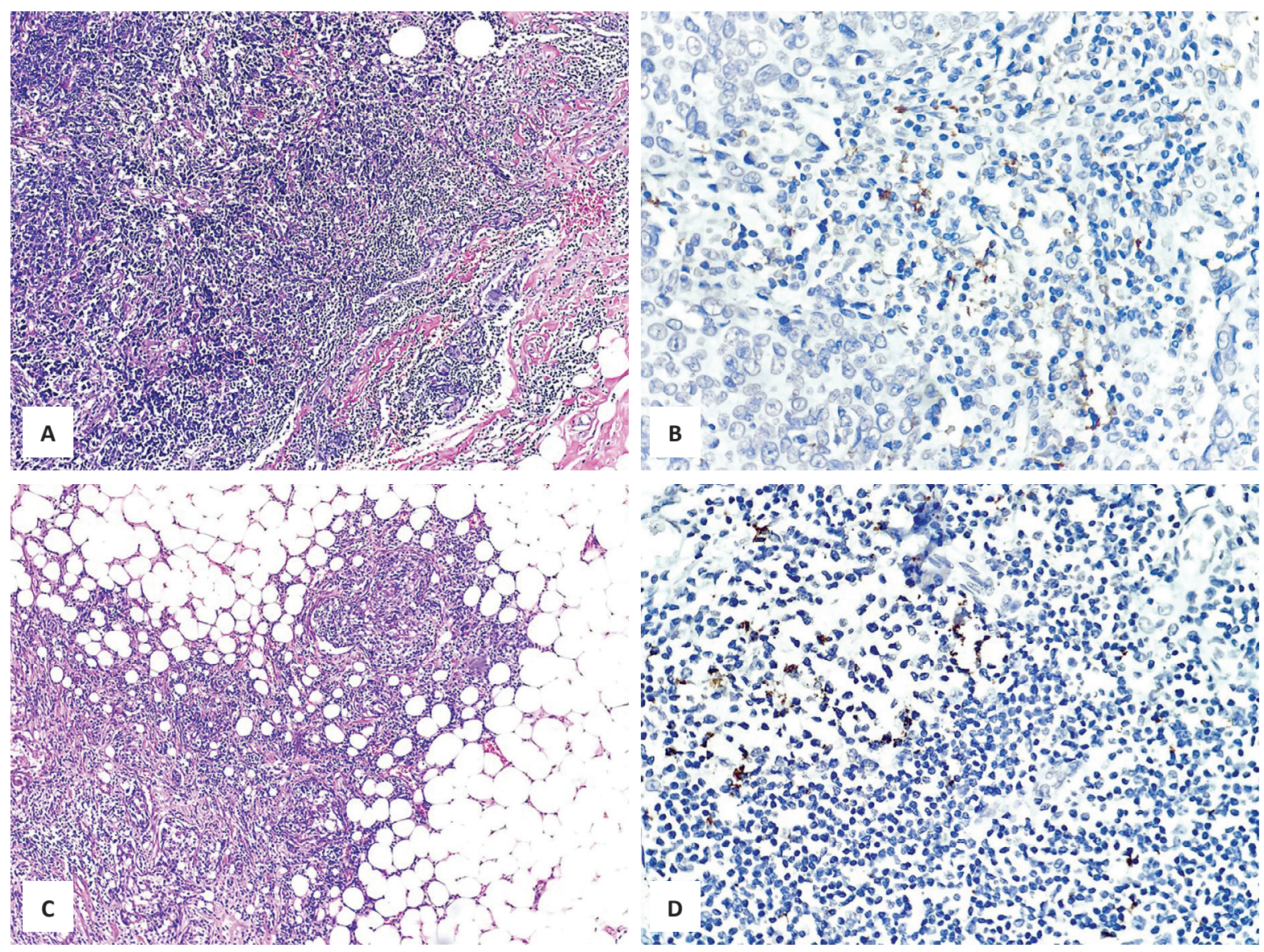

Figure 2. (A and C) Examples of cases with discordant TILs (H\&E, 10x) with (B and C) their corresponding discordant PD-L1 (SP142) stains (H\&E, 40x). Both are IDCA by histologic subtype, with (A) showing medullary pattern.

with heavy immune infiltrates. ${ }^{9}$ The kappa statistic must be interpreted in the proper clinical context, especially in the laboratory setting where high degree of precision is imperative. The results of the study showed only modest results, compared to the studies which produced good, if not substantial, reliability. ${ }^{9,10,24}$ The wide range of intraand interobserver agreement among readers brings into question the reproducibility of TILs in the local setting. Though standard protocols for TILs and PD-L1 evaluation were provided by the TILs Working Group and Roche Tissue Diagnostics, respectively, a standardized training for evaluation is warranted to improve the reproducibility of results.

Regardless of whether Filipino pathologists are ready to adapt this approach to breast specimens, breast pathology continues to move forward with the advancement of new treatment options. In fact, integrating PD-L1 and TIL scoring into routine practice would provide a comprehensive "immuno-oncological marker" for TNBC patients. It is of utmost importance to ensure its validity and reproducibility by optimizing the workflow from patient selection to tissue processing and standardized evaluation involving multiple disciplines. ${ }^{21}$

This study demonstrated variable intra and interobserver agreement for both TILs and PD-L1 expression. Although it is desirable to have strong to almost perfect agreement, the kappa and ICC values suggest additional room for improvement. The reasons for which may be due to the new adaptation of the SP142 assay in the institution and the limited experience of the pathologists in interpreting PD-L1 (SP142) immunostains despite taking the recommended course material. The variability of the results can also be due to the differences in pathologists' experience in signing out PD-L1 assays. The first reader has prior experience, albeit with a different clone (SP263) for non-small cell lung carcinoma, with a case load of more than 20 cases in the past two years while the second and third readers were naïve to PD-L1 interpretation. It is suggested that thresholds of each pathologist be realigned by reviewing discordant results to improve reliability. Methodology can be improved with further training and perhaps the use of digital pathology with standardized images for comparison. ${ }^{6}$ To improve reproducibility, whole slide images with the aid of computer-based image analysis and automated quantification were recommended. ${ }^{21}$ Further studies on PD-L1 expression may be done to investigate variables such as tissue samples, type of tumor, tumor heterogeneity which may affect consistency in interpretation. In light of the repercussions in management of patients who will undergo immune checkpoint inhibitor therapy, the following are suggested to achieve consistent results: regular training sessions for scoring, concurrences of equivocal results, and possible use of digital pathology as a medium in interpreting TILs and PD-L1 stains. 


\section{ACKNOWLEDGMENT}

The authors would like to acknowledge Dr. Kevin Elomina and Ms. Ma. Kristine Joy S. Calvario for performing the statistical analysis of the paper.

\section{STATEMENT OF AUTHORSHIP}

All authors fulfilled the ICMJE authorship criteria.

\section{AUTHOR DISCLOSURE}

The authors declared no conflict of interest.

\section{FUNDING SOURCE}

Roche (Philippines), Inc. donated PD-L1 SP142 test kits for the study, as requested by the principal investigator solely for research purposes. Roche (Philippines), Inc. had no influence on the conception, and outcome of the study.

\section{REFERENCES}

1. World Health Organization. International Agency for Research on Cancer. Globocan 2020 [cited 2020 Oct 31]. Available from https://gco.iarc.fr/today/data/ factsheets/populations/608-philippines-fact-sheets. pdf.

2. Foulkes WD, Smith IE, Reis-Filho JS. Triple Negative breast cancer. N Engl J Med. 2010;363(20): 1938-48. PMID: 21067385. https://doi.org/10.1056/ NEJMra1001389.

3. Loi S, Michiels S, Salgado R, et al. Tumor infiltrating lymphocytes are prognostic in triple negative breast cancer and predictive for trastuzumab benefit in early breast cancer: results from the FinHER trial. Ann Oncol. 2014;25(8):1544-50. PMID: 24608200. https://doi.org/10.1093/annonc/mdu 112.

4. Schütz F, Stefanovic S, Mayer L, Von Au A, Domschke C, Sohn C. PD-1/PD-L1 pathway in breast cancer. Oncol Res Treat. 2017;40(5):294-7. PMID: 28346916. https://doi.org/10.1159/000464353.

5. Paver EC, Cooper WA, Colebatch AJ, et al. Programmed death ligand-1 (PD-L1) as a predictive marker for immunotherapy in solid tumours: a guide to immunohistochemistry implementation and interpretation. Pathology. 2021;53(2):141-56. PMID: 33388161. https://doi.org/10.1016/j.pathol. 2020.10.007.

6. Salgado R, Denkert C, Demaria S, et al. The evaluation of tumor-infiltrating lymphocytes (TILS) in breast cancer: recommendations by an International TILS Working Group 2014. Ann Oncol. 2015;26(2): 259-71. PMID: 25214542. PMCID: PMC6267863. https://doi.org/10.1093/annonc/mdu450.

7. Rakha E, Allison K, Ellis I, Horii R, Masuda S, PenaultLlorca F, et al. WHO Classification of tumours. Breast tumours, 5th ed, vol. 2. Lyon; 2019. Available from https://publications.iarc.fr/581.

8. Roche. VENTANA PD-L1 (SP142) Assay interpretation guide for triple-negative breast carcinoma (TNBC); 2019.

9. Swisher SK, Wu Y, Castaneda CA, et al. Interobserver agreement between pathologists assessing tumor- infiltrating lymphocytes (TILs) in breast cancer using methodology proposed by the International TILs Working Group. Ann Surg Oncol. 2016;23(7):2242-8. PMID: 26965699. https://doi.org/10.1245/s10434-0165173-8.

10. Tramm T, Di Caterino $\mathrm{T}$, Jylling AMB, et al. Standardized assessment of tumor-infiltrating lymphocytes in breast cancer: an evaluation of inter-observer agreement between pathologists. Acta Oncol (Madr). 2018;57(1):90-4. PMID: 29168428. https://doi.org/ 10.1080/0284186X.2017.1403040.

11. McHugh ML. Lessons in biostatistics interrater reliability : the kappa statistic. Biochem Med (Zagreb). 2012;22(3):276-82. PMID: 23092060. PMCID: PMC3900052.

12. Koo TK, Li MY. A Guideline of selecting and reporting intraclass correlation coefficients for reliability research. J Chiropr Med. 2016;15(2): 155-63. PMID: 27330520. PMCID: PMC4913118. https://doi.org/10.1016/j.jcm.2016.02.012.

13. Kumar P, Aggarwal R. An overview of triple-negative breast cancer. Arch Gynecol Obstet. 2016;293(2):24769. PMID: 26341644. https://doi.org/10.1007/s00404015-3859-y.

14. Schmid P, Rugo HS, Adams S, et al. Atezolizumab plus nab-paclitaxel as first-line treatment for unresectable, locally advanced or metastatic triple-negative breast cancer (IMpassion 130): updated efficacy results from a randomised, double-blind, placebo-controlled, phase 3 trial. Lancet Oncol. 2020;21(1):44-59. PMID: 31786121. https://doi.org/10.1016/S14702045(19)30689-8.

15. AiErken NJ, Shi HJ, Zhou Y, et al. High PD-L1 expression is closely associated with tumor-infiltrating lymphocytes and leads to good clinical outcomes in Chinese triple negative breast cancer patients. Int J Biol Sci. 2017;13(9):1172-9. PMID: 29104508. PMCID: PMC5666332. https://doi.org/10.7150/ ijbs.20868.

16. Lee HT, Lee JY, Lim H, et al. Molecular mechanism of PD-1/PD-L1 blockade via anti-PD-L1 antibodies atezolizumaband durvalumab. Sci Rep. 2017;7(1):5532. PMID: 28717238. PMCID: PMC5514103. https://doi. org/10.1038/s41598-017-06002-8.

17. Zhang M, Sun H, Zhao S, et al. Expression of PDL1 and prognosis in breast cancer: a meta-analysis. Oncotarget. 2017;8(19):31347-54. PMID: 28430626. PMCID: PMC5458212. https://doi.org/10.18632/ oncotarget.15532.

18. Chaher N, Qualls C, Joste N, et al. The combined presence of $\mathrm{CD} 20+\mathrm{B}$ cells and PD-L1+ tumor infiltrating lymphocytes in inflammatory breast cancer is prognostic of improved patient outcome. Breast Cancer Res Treat. 2018;171(2):273-82. PMID: 29858752. PMCID: PMC6173952. https://doi. org/10.1007/s10549-018-4834-7.

19. García-Teijido P, Cabal ML, Peláez Fernández I, Fernández Pérez Y. Tumor-infiltrating lymphocytes in triple negative breast cancer: the future of immune targeting. Clin Med Insights Oncol. 2016;10(Suppl 1):31-9. PMID: 27081325. PMCID: PMC4822722. https://doi.org/10.4137/CMO.S34540

20. Saleh R, Taha RZ, Nair VS, Alajez NM, Elkord E. PDL1 blockade by atezolizumab downregulates signaling 
pathways associated with tumor growth, metastasis, and hypoxia in human triple negative breast cancer. Cancers (Basel). 2019;11(8):1050. PMID: 31349612. PMCID: PMC6721435. https://doi.org/10.3390/ cancers11081050.

21. Gonzalez-Ericsson PI, Stovgaard ES, Sua LF, et al. The path to a better biomarker: application of a risk management framework for the implementation of PD-L1 and TILs as immuno-oncology biomarkers in breast cancer clinical trials and daily practice. J Pathol. 2020;250(5):667-84. PMID: 32129476. https://doi. org/10.1002/path.5406.

22. Reisenbichler ES, Han G, Bellizzi A, et al. Prospective multi-institutional evaluation of pathologist assessment of PD-L1 assays for patient selection in triple negative breast cancer. Mod Pathol. 2020;33(9):1746-52. PMID: 32300181. PMCID: PMC8366569. https:// doi.org/10.1038/s41379-020-0544-x.
23. Downes MR, Slodkowska E, Katabi N, Jungbluth $\mathrm{AA}, \mathrm{Xu}$ B. Inter- and intraobserver agreement of programmed death ligand 1 scoring in head and neck squamous cell carcinoma, urothelial carcinoma and breast carcinoma. Histopathology. 2020;76(2): 191-200. PMID: 31243779. PMCID: PMC6917963. https://doi.org/10.1111/his.13946.

24. Kojima YA, Wang X, Sun H, Compton F, Covinsky M, Zhang S. Reproducible evaluation of tumor-infiltrating lymphocytes (TILs) using the recommendations of International TILs Working Group 2014. Ann Diagn Pathol. 2018;35:77-9. PMID: 29886396. https://doi. org/10.1016/j.anndiagpath.2018.05.007.

\section{EDITORIAL COMMENT}

In August 27, 2021, Roche announced their decision to voluntarily withdraw the US accelerated approval for Tecentriq ${ }^{\circledR}$ (atezolizumab) in combination with chemotherapy (Abraxane ${ }^{\circledR}$, albumin-bound paclitaxel) for the treatment of adults with unresectable locally advanced/metastatic triple-negative breast cancer whose tumors express PD-L1. The drug has other approved indications for other types of cancer. Ventana PD-L1 (SP142) assay is an approved companion diagnostic device for selecting TNBC candidates for atezolizumab treatment. Our peer reviewer pointed out the effect of the withdrawal of the drug from the market as a treatment for TNBC to the significance of the SP142 companion diagnostic assay. Philippine Journal of Pathology, however, believes that the paper still deserves to be published. It retains its value as an academic paper for Filipino pathologists, pathologists in general, to learn much from in terms of mutation detecting assays for candidate screening, cancer prognostication, treatment monitoring, et cetera. In a post-review communication, the authors state that "though atezolizumab was withdrawn from the market, the SP-142 study demonstrates variable thresholds of pathologists and how differences in experience can affect interpretation. Addressing these gaps in these diagnostic tools may help in terms of quality improvement in the practice of pathology." Further, they add that "the SP-142 assay may not (no longer) be used for TNBCs but still has utility for other malignancies, such as NSCLC (non-small cell lung carcinoma), though with different cut-offs and cells of interest. This exposes the need for training of pathologists and adjustments of thresholds in interpreting PD-Ll expression, especially since these biomarkers are new to the country with limited accessibility. This applies to other clones and brands currently available in the market and those in future development."

\section{References:}

1. FDA approves atezolizumab for PD-L1 positive unresectable locally advanced or metastatic triple-negative breast cancer. News release. FDA. March 8, 2019. Accessed August 27, 2021. https://bit.ly/374kYXE

2. Roche provides update on Tecentriq US indication for PD-L1-positive, metastatic triple-negative breast cancer. News release. August 27, 2021. Accessed August 27, 2021. https://bit.ly/3zvwism

3. Roche provides update on phase III study of Tecentriq in combination with paclitaxel for people with metastatic triple-negative breast cancer. News release. Roche. August 6, 2020. Accessed August 27, 2021. https://bit.ly/3knjlEk

4. A study of atezolizumab and paclitaxel versus placebo and paclitaxel in participants with previously untreated locally advanced or metastatic triple negative breast cancer (TNBC) (IMpassion131). ClinicalTrials.gov. Updated July 7, 2020. Accessed August 27, 2021. https://clinicaltrials.gov/ct2/show/NCT03125902

Disclaimer: This journal is OPEN ACCESS, providing immediate access to its content on the principle that making research freely available to the public supports a greater global exchange of knowledge. As a requirement for submission to the PJP, all authors have accomplished an AUTHOR FORM, which declares that the ICMJE criteria for authorship have been met by each author listed, that the article represents original material, has not been published, accepted for publication in other journals, or concurrently submitted to other journals, and that all funding and conflicts of interest have been declared. Consent forms have been secured for the publication of information about patients or cases; otherwise, authors have declared that all means have been exhausted for securing consent. 